\title{
Final Report for SSAA Grant NA26170
}

\author{
DE-FG52-06NA26170 \\ Quantum Simulations for Dense Matter \\ PIs: David M. Ceperley and Richard M. Martin: \\ University of Illinois Urbana-Champaign
}

Date of report: June 1, 2010

Period of grant: May 2006 - April 2010

\section{Executive Summary:}

High pressure systems are important, for example, to understand the interiors of giant planets (Jupiter and Saturn), for experiments at NIF (the National Ignition Facility at Livermore) related to inertially confined fusion and for other interests of DOE. In this project we are developing innovative simulation methods (Quantum Monte Carlo methods) to allow more accurate calculation of properties of systems under extreme conditions of pressure and temperature. These methods can use the power of current day supercomputers made of very many processors, starting from the basic equations of physics to model quantum phenomena important at the microscopic scale. During the grant period, we have settled two important questions of the physics of hydrogen and helium under extreme conditions. We have found the pressures and temperatures when hydrogen and helium mix together; this is important to understand the difference of the interiors of the planets Jupiter and Saturn. Secondly, we have shown that there exists a sharp transition as a function of pressure between molecular and atomic liquid hydrogen at temperatures below $2000 \mathrm{~K}$. This prediction can be confirmed with high pressure experiments.

\section{Accomplishments:}

The main goal of the research under this grant was to develop the coupled electron ion Monte Carlo method to produce reliable thermodynamic predictions of systems at high temperature and density. Most of the effort 
has been to develop highly parallel efficient codes, improved methodology, and applications to the equation of state of warm dense hydrogen using the Coupled Electron-Ion Monte Carlo method. We have also developed methods to calculate the conductivity of dense hydrogen. In the last few years, we have done important calculations using these methods on high end computers. Full details are given in the publications listed at the end of this report in particular, refs $4,8,9$, and 10.

Our principal finding has concerned the atomic-molecular transition in dense liquid hydrogen. A first-order liquid-liquid phase transition in highpressure hydrogen between molecular and atomic fluid phases has been predicted in computer simulations using ab initio molecular dynamics approaches. However, some experiments indicate that molecular dissociation may occur through a continuous crossover rather than a firstorder transition. We studied the nature of molecular dissociation in fluid hydrogen using the CEIMC method and using DFT simulations and found a weakly first-order liquid-liquid phase transition for temperatures lower than $2000 \mathrm{~K}$. This paper has been recently accepted for publication in PNAS (ref 10).

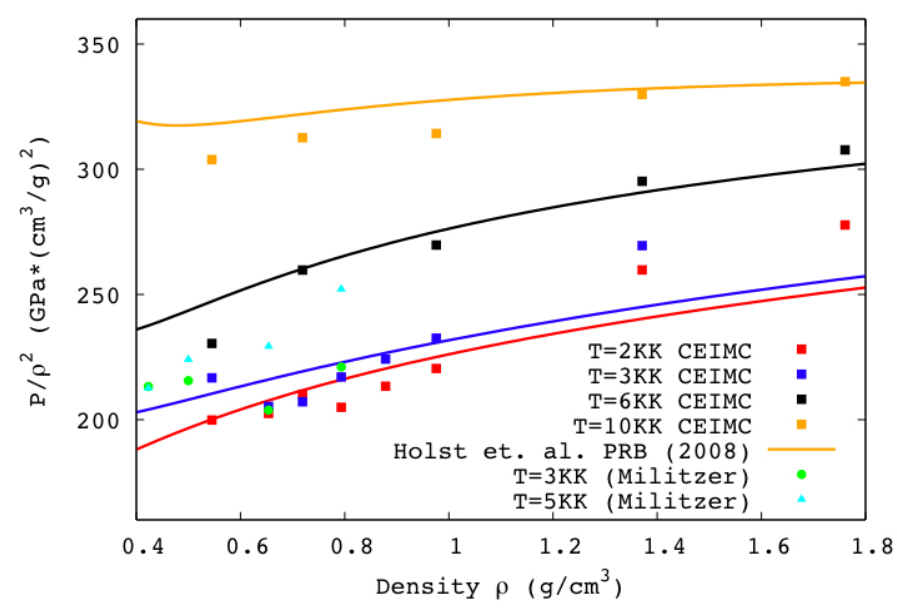

Figure 1 Comparison of the pressures calculated using CEIMC vs. AIMD calculations by Holst et al.
We applied CEIMC to metallic atomic hydrogen at low temperatures $(T<1000 \mathrm{~K})$. Estimates were made of the melting temperatures of b.c.c. hydrogen. We have used CEIMC to calculate the equation of state of hydrogen for temperatures from $2000 \mathrm{~K}$ to $10000 \mathrm{~K}$ and densities in units of $r_{s}$ from 2.0 to 1.15. Comparisons of the pressures with those of the recent AIMD

calculations of Holst et al are shown in Fig. 1. Clearly the overall trends in the results from the two methods are in agreement; however, there are interesting differences we are analyzing.

We have completed EOS calculations on hydrogen systems of 32 and 54 protons. Simulations with 108 protons are in progress on computers at Oak Ridge National Laboratory and at the NCSA (the National Center for 
Supercomputing Applications). Extensive testing has been performed to understand and correctly control all the parameters in the quantum Monte Carlo simulations. From these results, we have obtained accurate pressures and energies which will be used to produce free energies and other thermodynamic information for hydrogen under conditions relevant to the interiors of the giant planets as well as the NIF experiments. This is for pressures between $60 \mathrm{GPa}$ and $600 \mathrm{GPa}$ and temperatures from $300 \mathrm{~K}$ to $6000 \mathrm{~K}$. We have estimated finite size corrections by a combination of size extrapolation and other correction techniques based on the small-k behavior of the static structure factor. We have also estimated the importance of the protonic zero point motion in the various phases and regimes. With current funding, we feel that we can make a benchmark quality EOS for dense hydrogen, of enduring value. The goal set by the needs of planetary models is an accuracy of $1 \%$ in the pressure. The differences between the methods are still larger than that; but with improved methodology, achievement of this goal is in sight. (see ref 8)

Metal-insulator transition with density variation

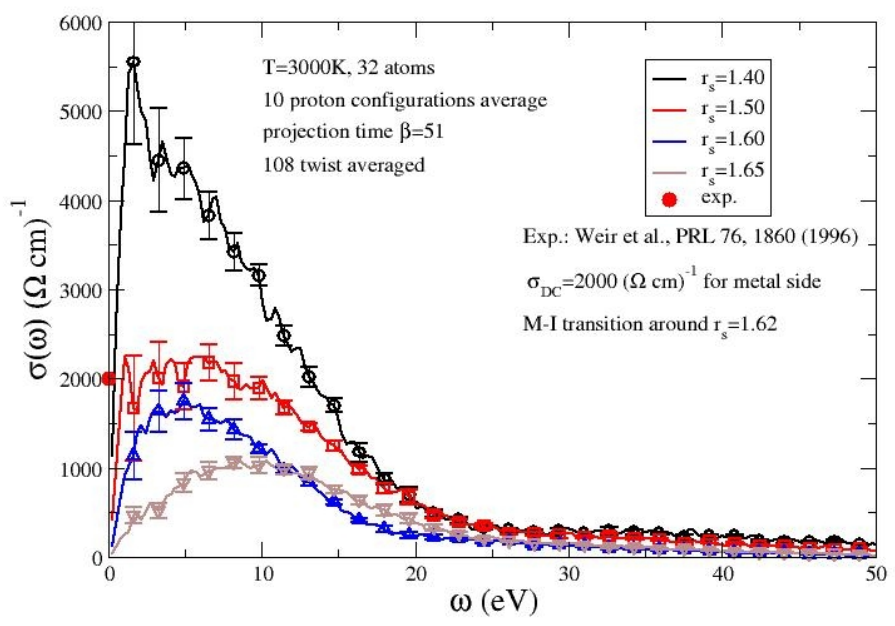

We also used

CEIMC to calculate the electrical conductivity for liquid hydrogen under high pressure. The method uses correlated Monte Carlo sampling to calculate a lowest many-body states and currentcurrent matrix elements, which are summed over in the many-body Kubo-Greenwood formula to give ac electrical conductivity. This is the first time such a calculation has been done for a continuum many-body system using quantum Monte Carlo techniques. In the limit of zero frequency, we obtain the dc conductivity, which can be compared to the experimentally measured ones. The extrapolated dc conductivity at temperature $3000 \mathrm{~K}$ for several densities shows a liquid semiconductor to liquid metal transition under high pressure. Our results are in good agreement with shock-wave experiments. Earlier DFT dc conductivity calculations give a value of about 6 times the experimental value. It is worth noting that DFT calculation of AC conductivity based on the one-body Kubo-Green-wood 
formula usually overestimates the dc conductivity. Recent molecular dynamics (MD) simulations show better agreements with the experimental conductivity and reflectivity data, but nonetheless, these simulations still have the independent particle approximation. It is quite possible that these dc conductivity data from MD still overestimate the real value, making the liquid hydrogen more "metallic" than it should be. Our ac conductivity results at $3000 \mathrm{~K}$ show a liquid semiconductor to liquid metal transition around $r_{s}=1.50$ with a dc conductivity of $2000(\Omega \mathrm{cm})^{-1}$, in reasonable agreement with the experimental dc conductivity with a transition density of about $r_{s}=1.62$ at $3000 \mathrm{~K}$. See ref 9 .

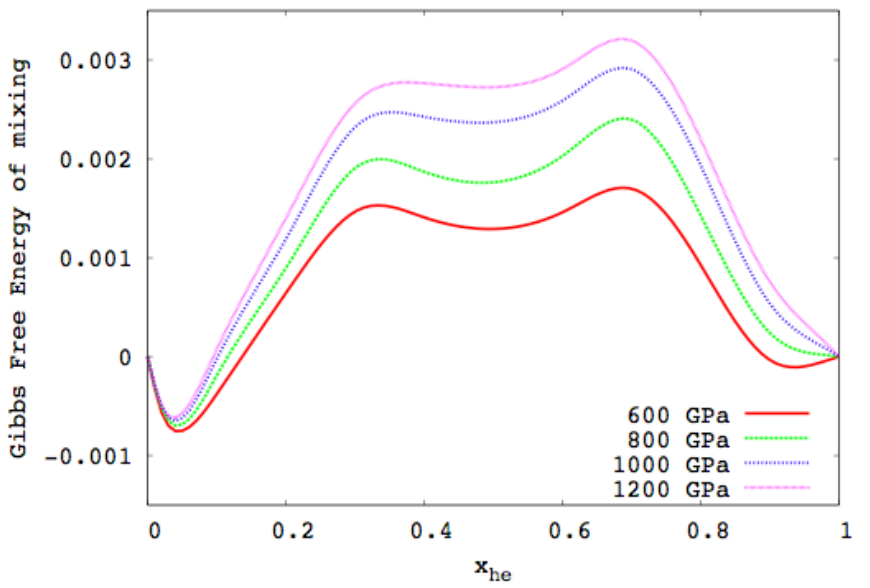

Figure 3. The Gibbs free energy of mixing at $\mathrm{T}=6000 \mathrm{~K}$ as a function of pressure and helium concentration.
We have performed calculations of hydrogen-helium mixtures to calculate the temperature, as a function of pressure, at which helium becomes insoluble in dense metallic hydrogen. Miguel Morales (Illinois graduate student) was awarded a DOE-NNSA Stewardship Sciences Graduate

Fellowship. He spent the summer of 2008 at LLNL as an intern with the Quantum Simulations Group and worked on this problem while at Livermore. At LLNL, he performed AIMD calculations of the free energy of mixing as a function of temperature and pressure and found a demixed phase. The figure above shows the Gibbs free energy of mixing as a function of pressure and helium concentration. Morales et al. performed an extensive study of the equation of state of the mixture as a function of density, temperature, and composition and, together with a few different thermodynamic integration techniques, calculated the Gibbs free energy of mixing using coupling constant integration methods; the immiscibility temperature could be directly estimated without the need to resort to approximations to the entropy of mixing. The structural properties of the liquid as a function of composition were also studied to understand how 
helium stabilizes the hydrogen bonds even at pressures above $10 \mathrm{Mbar}$. A paper on this appeared in PNAS in 2009 (ref. 7)

Concerning method developments, we invented a new sampling algorithm in the framework of the reptation quantum Monte Carlo method chosen to sample the electronic degrees of freedom, thereby improving its efficiency. We wrote a detailed description of the method (ref. 4). Many-body trial wave functions are the key ingredient for accurate Quantum Monte Carlo estimates of total electronic energies in many-electron systems. In the CEIMC method, there is a trade-off between the accuracy of the trial function and the efficiency of its evaluation. We report recent progress in trial wave functions for metallic hydrogen implemented in the CEIMC method. We described and characterized several types of trial functions of increasing complexity in the range of the coupling parameter $1.0<r_{s}$ $<1.55$. We reported comparisons of wave function for disordered protonic configurations and preliminary results for thermal averages. (ref 6)

During the grant period we have improved several aspects of our parallel Coupled Electron Ion Monte Carlo code (bopimc). In particular, we have incorporated backflow correlations, implemented faster density functional solvers and improved its scalability. We have ported the code to the Abe computer at NCSA and to the Jaguar computer at ORNL and are performing calculations on those computers as well as at LLNL. We were granted time for this project at NCSA (NSF teragrid program) and at Oak Ridge National Lab (DOE INCITE program).

During the grant period, D. Ceperley has given about 15 invited lectures at various conferences and institutions, including a CASC talk at Livermore, at ORNL, and an invited talk at the March APS meeting, and he attended the annual NNSA meeting in Washington. C. Pierleoni and M. Morales have also given invited talks on this work at international meetings, such as CCP2007 (Brussels), RBMBPT14 (Barcelona) and SCCS08 (Camerino). 


\section{Publications:}

1. Holzmann, M., C. Pierleoni, and D. M. Ceperley, Coupled Electron Ion Monte Carlo Calculations of Atomic Hydrogen*, CCP2004 conference proceedings, Elsevier Science, cond-mat/0410530.

2. Pierleoni, C. and D. M. Ceperley, Computational Methods in Coupled Electron-Ion Monte Carlo, ChemPhysChem, 6, 1 (2005); physics/0501013.

3. Delaney, K., C. Pierleoni and D. M. Ceperley, Quantum Monte Carlo Simulation of the High Pressure Molecular-Atomic Transition in Fluid Hydrogen, Phys. Rev. Letts. 97 , 235702: 1-4(2006); cond-mat/0603750.

4. Pierleoni, C. and D. M. Ceperley, The Coupled Electron-Ion Monte Carlo Method, Computer Simulations in Condensed Matter Systems: From Materials to Chemical Biology, eds. M. Ferrario, G. Ciccotti, K. Binder, Lecture Notes in Physics, 703 , pgs. 641-683, Springer Berlin Heidelberg , 2006; physics/0510254.

5. Pierleoni, C, Delaney, K. T, Morales, M. A., Ceperley, D. M., Holzmann, M., Progress in Coupled Electron-Ion Monte Carlo Simulations of HighPressure Hydrogen, Recent Progress in Many-Body Theory, Series on Advances in Quantum Many-Body Theory- Vol. 11, eds. J. Boronat, G. Astrakharchik, F. Mazzanti, World Scientific, pgs. 217-232, (2008).

6. Pierleoni, C, Delaney, K. T, Morales, M. A., Ceperley, D. M., Holzmann, M. Trial wave functions for High Pressure Metallic Hydrogen, Computer Physics Communications 179, 89-97 (2008); physics/0712.0161.

7. M. A. Morales, E. Schwegler, D. Ceperley, C. Pierleoni, S. Hamel, and K. Caspersen, Phase separation in hydrogen-helium mixtures at Mbar pressures, Proceedings of the National Academy of Sciences 106, 13241329 (2009).

8. M. A. Morales, C. Pierleoni, D. M. Ceperley, Equation of state of metallic hydrogen from Coupled Electron-Ion Monte Carlo simulations, Phys. Rev. E 81, 021202:1-9; arXiv:0906.1594.

9. Fei Lin, Miguel A. Morales, Kris T. Delaney, Carlo Pierleoni, Richard M. Martin, and D. M. Ceperley, Electrical Conductivity of High-Pressure Liquid Hydrogen by Quantum Monte Carlo Methods, Phys. Rev. Letts. 103, 256401:1-4 (2009), arXiv:0909.2248.

10. M. Morales, E. Schwegler, C. Pierleoni and D. Ceperley, "Evidence for a first order liquid-liquid transition in high pressure hydrogen from ab-initio simulations" in press, Proceedings of the National Academy of Sciences, 2010.

The following papers were written by Fei Lin (the PD supported by this grant). They do not directly concern warm dense matter, but are on the topic of his $\mathrm{PhD}$ work, correlation in fullerenes. 
11. Fei Lin, E. Sorensen, C. Kallin and J. Berlinsky, "Single-particle Excitation Spectra of C60 Molecules and Monolayers", Phys. Rev. B 75, 075112 (2007),

12. Fei Lin, E. Sorensen, C. Kallin and J. Berlinsky, "Strong Correlation Effects in the Fullerene C20", Phys. Rev. B 76, 033414 (2007),

13. Fei Lin, E. Sorensen, C. Kallin and J. Berlinsky "Extended Hubbard model on a C20 molecule", J. Phys.: Condens. Matter 19, 456206 (2007),

14. Fei Lin and E. Sorensen, "Estimates of effective Hubbard model parameters for C20 isomers", Phys. Rev. B 78, 085435 (2008),

15. Fei Lin, E. Sorensen, C. Kallin and J. Berlinsky, "C20, the Smallest Fullerene", invited book chapter in "Handbook of Nanophysics", Edited by Klaus Sattler, Taylor \& Francis Publisher (CRC Press) (2010), 